\title{
Le sens objectif des faits sociaux
}

Problèmes sémantiques de la sociologie compréhensive

\section{Patrick Pharo}

\section{CpenEdition}

\section{Journals}

Édition électronique

URL : https://journals.openedition.org/ress/679

DOI : $10.4000 /$ ress. 679

ISSN : 1663-4446

Éditeur

Librairie Droz

\section{Édition imprimée}

Date de publication : 1 décembre 2000

Pagination : 139-157

ISBN : 2-600-00481-5

ISSN : 0048-8046

\section{Référence électronique}

Patrick Pharo, "Le sens objectif des faits sociaux 》, Revue européenne des sciences sociales [En ligne], XXXVIII-119 | 2000, mis en ligne le 15 décembre 2009, consulté le 28 juin 2022. URL : http:// journals.openedition.org/ress/679; DOI : https://doi.org/10.4000/ress.679 


\section{Patrick PHARO}

\section{LE SENS OBJECTIF DES FAITS SOCIAUX problèmes sémantiques de la sociologie compréhensive}

L'objectivation des faits sociaux repose-t-elle uniquement sur l'observation de données perceptuelles, c'est-à-dire physiques et comportementales, ou doit-elle aussi se fonder sur des données logico-sémantiques relatives au sens de ces faits pour les acteurs sociaux et pour les observateurs? On pourrait dire que toute la recherche empirique en sociologie et en histoire, qui admet généralement le rôle causal des motifs, des idéologies ou des valeurs, qui ne sont au fond que des faits sémantiques $^{1}$ et non pas des choses physiques, plaide pour le second terme de l'alternative. Or, comme je voudrais le montrer dans la suite de cet article, l'hypothèse d'une objectivité et d'un rôle causal spécifiques des faits sémantiques dans la vie sociale se heurte à des difficultés épistémologiques si importantes qu'on pourrait être tenté de l'abandonner ou, au moins, de chercher à réduire les faits qui la concernent à des données physiologiques ou strictement comportementales. Mon but sera au contraire de suggérer qu'il y a de bonnes raisons de ne pas abandonner ou d'affaiblir cette hypothèse, mais qu'il faut plutôt essayer de la clarifier et de la rendre compatible avec les données des sciences contemporaines de la nature, dont la sociologie, jusqu'à preuve du contraire, fait encore partie.

La meilleure façon, me semble-t-il, d'accomplir ce programme, est de préciser le rôle des contenus logico-sémantiques, tels que le sens et les pensées, dans l'émergence des faits sociaux. C'est ce que tente de faire le présent article, en proposant de traiter ces contenus comme la manifestation d'un ordre logique, et non pas empirique, qui fait peser un certain nombre de contraintes à la fois sur l'action sociale et sur sa description sociologique ${ }^{2}$. Cette démarche s'inscrit bien sûr dans la tradition de la sociologie compréhensive, dont les problèmes et les critiques, notamment celles de Parsons, seront examinés dans la deuxième section. Mais auparavant, je présenterai dans une première section les principaux aspects de l'ontologie sociale de Durkheim afin de préciser le cadre général du problème de l'objectivation sociologique du sens. Enfin, la troisième section situera le pro-

On parle aussi habituellement en anthropologie et en sociologie de faits symboliques, mais «symbole» est un terme ambigu car il inclut à la fois la matérialité du signe et son contenu de signification. Pour cerner la spécificité du contenu des signes, on doit donc préférer l'adjectif sémantique, qui renvoie à un usage linguistique - la sémantique lexicale -, aussi bien qu'à un usage logique, relatif au sens et à la valeur de vérité des expressions d'un langage.

2 L'opposition signalée ici entre ordre logique et faits empiriques correspond à l'opposition philosophique classique des faits de raison et des faits d'expérience, et non pas à celle, plus moderne, des actions rationnelles (ou logiques) et irrationnelles (ou illogiques) que l'on trouve dans la tradition de la sociologie de la rationalité. 
blème du sens objectif des faits sociaux par rapport au programme contemporain de naturalisation de l'esprit avant d'expliciter quelques critères susceptibles d'attester l'indépendance logique du sens et des pensées dans l'accomplissement des faits sociaux.

\section{LES FAITS SOCIAUX COMME CHOSES PENSÉES ET PENSANTES}

Comme on le sait, Durkheim recommandait de traiter les faits sociaux comme des choses, car il considérait que leurs effets sur les êtres humains s'exercent de façon strictement externe et que c'est précisément ce caractère de coercition externe, indépendant de tout «procédé d'analyse mentale» ${ }^{3}$, qui fait la force et la réalité des faits sociaux. Un partisan de la sociologie compréhensive pourrait évidemment objecter que si les choses sociales sont des choses, ce doit être probablement dans un sens assez particulier, car il est difficile de les identifier aux seules choses qui sont indiscutablement des choses, comme les choses physiques. Et il pourrait encore ajouter, dans le même ordre d'idées, que c'est précisément parce que les choses sociales sont, par leur sens ou leur signification ${ }^{4}$, «compénétrables à l'intelligence» qu'elles exercent leurs effets sur les consciences et sur les actes, et qu'il est donc difficile, dans ces conditions, de les traiter comme des choses strictement externes. Imaginons par exemple un extra-terrestre qui observerait la terre et ses habitants sans connaître ni chercher à connaître leur langage et leurs pensées, que pourrait-il apprendre d'eux en les traitant comme des choses simpliciter? En réalité, une connaissance des sociétés humaines construite indépendamment de toute tentative de compréhension interne ne serait qu'une sorte d'écologie béhavioriste, classique ou plus moderne, intégrant, comme cela se fait aujourd'hui, les fonctions d'adaptation cognitive, mais ce ne serait pas vraiment une sociologie.

Cependant, et fort heureusement pour la sociologie, Durkheim n'a jamais envisagé les choses sociales d'une façon aussi sommaire et il s'est au contraire élevé de façon virulente et convaincante contre toute forme de réductionnisme appliqué aux faits sociaux ${ }^{5}$. Ainsi, lorsqu'il tente de préciser la spécificité des faits sociaux, il remarque que n'importe quel phénomène qui se passe dans la société peut avoir un intérêt social: boire, manger, dormir, raisonner ${ }^{6}$. Mais ce qui, selon

3 «Est chose, écrit Durkheim, tout objet de connaissance qui n'est pas naturellement compénétrable à l'intelligence, tout ce dont nous ne pouvons nous faire une notion adéquate par un simple procédé d'analyse mentale, tout ce que l'esprit ne peut arriver à comprendre qu'à condition de sortir de lui-même, par voie d'observations et d'expérimentations, en passant progressivement des caractères les plus extérieurs et les plus immédiatement accessibles aux moins visibles et aux plus profonds », Les règles de la méthode sociologique, Paris, PUF 1967, éd. orig. 1895, p. XIII.

4 Dans le présent, texte, je n'introduis pas de différence systématique entre ces deux mots, bien que cela se fasse parfois, notamment pour traduire l'allemand Sinn (sens) et Bedeutung (signification), le deuxième terme renvoyant plutôt à l'objet signifié tandis que le premier renvoie à son mode de présentation dans l'esprit.

5 Et en particulier dans Représentations individuelles et représentations collectives, in Sociologie et philosophie, Paris, PUF, 1974, éd. orig. 1898.

$6 \quad$ Les règles de la méthode sociologique, op. cit., p. 3. 
Durkheim, constitue spécifiquement le fait social, et le différencie ultimement des faits physiologiques et psychologiques, ce sont des devoirs de droit et de mœurs, des systèmes de signes et de monnaie, des manières d'agir et des courants sociaux qui existent en-dehors de la conscience individuelle et s'imposent à elle sous peine de sanctions collectives. De là découlent la définition des faits sociaux comme manières de faire susceptibles d'exercer sur l'individu une contrainte extérieure, mais aussi l'idée d'une «espèce nouvelle» qui ne se confondrait ni avec les phénomènes organiques, car elle n'est constituée que de représentations et d'actions, ni avec les phénomènes psychiques, car elle est extérieure à la conscience individuelle (ibid., p. 5). On aboutit ainsi à cette fameuse réalité sociale sui generis dont on trouve plusieurs mentions dans l'œuvre de Durkheim, notamment dans l'article Représentations individuelles et représentations collectives et qui, loin d'être une chose strictement physique, est plutôt une réalité d'ordre spirituel. Le «chosisme» de Durkheim est donc tout le contraire d'un béhaviorisme ou d'un physicalisme, puisqu'il vise surtout à préserver la sociologie de tout réductionnisme, psychologique ou physiologique.

$\mathrm{Au}$ demeurant, on voit mal comment la sociologie morale qui joue un si grand rôle dans toute l'œuvre de Durkheim, aurait pu se construire sans analyse des significations et sans une «compénétration par l'intelligence» des faits sociaux. Et de fait, n'étant guère tenté par le béhaviorisme, Durkheim ne s'est jamais privé d'analyser les faits sociaux du point de vue de leur signification interne. Sa notion d'institutions incluait d'ailleurs, en plus des modes de conduite institués par la collectivité, les croyances ${ }^{7}$, qui sont des faits cognitifs dont la matérialité est loin d'être évidente. De même, lorsque Durkheim présente la société ou la conscience collective comme une force qui s'exerce de l'extérieur sur les individus, il est peu probable qu'il croie avoir découvert une nouvelle entité ou une sorte de nouvelle force naturelle que l'on pourrait ajouter aux différentes forces physiques déjà connues. En réalité, le contenu concret de la force sociale qu'il envisage ne peut se révéler que dans des « choses » telles que les sanctions diffuses ou les sanctions organisées ou encore dans des courants sociaux, autrement dit dans des pratiques humaines qui ne sont ce qu'elles sont et n'entraînent leurs conséquences que par le sens qu'elles véhiculent. C'est donc bien en tant que choses «symboliques », comme on a coutume de dire, et non pas seulement «poussantes» ou «électrisantes » ou «atomisantes» que s'exerce, selon Durkheim, la force des faits sociaux.

Mais alors, si les faits sociaux ne sont pas des choses au sens strict, on peut se demander ce qu'ils sont en réalité. La réponse que je vais tenter d'argumenter dans la suite de ce texte et qui, comme on vient de le voir, est déjà en partie présente chez Durkheim, est que les faits sociaux sont bien des choses, mais des choses auxquelles se mêlent des pensées humaines, autrement dit des faits dont l'objectivité et l'efficience causale dépendent d'un contenu de pensée soumis à certaines contraintes logico-sémantiques s'exerçant sur les agents aussi bien que sur l'observateur - d'où l'expression de faits sémantiques sous laquelle on peut aussi les désigner. Les faits sociaux ne se réalisent évidemment que sous la forme d'êtres concrets, c'est-à-dire existants dans le temps et l'espace, comme des êtres

7 Les règles de la méthode sociologique, op. cit., p. XXII. 
humains, des actions ou des objets physiques, mais ils intègrent aussi de façon constitutive les pensées de ces êtres relatives à eux-mêmes, à leurs actions et aux objets qui les entourent. Et c'est parce que le statut spécifique des faits sociaux tient précisément à ce caractère «compénétrable» à l'intelligence, c'est-à-dire compréhensible du point de vue du sens intrinsèque, que leur causalité ne relève pas uniquement de la causalité physique, mais d'une causalité par le sens ou les raisons $^{8}$. Cette façon d'envisager les choses s'inscrit évidemment dans la continuité directe du programme wébérien de sociologie compréhensive et dans ses prolongements schutziens, en particulier l'idée que les typifications sociologiques ont un caractère de second ordre du fait qu'elles se rapportent toujours à des typifications sociales déjà existantes ${ }^{9}$.

Cependant, avant de revenir plus en détail dans la prochaine section sur les hypothèses de la sociologie compréhensive et les difficultés qu'elles soulèvent, il peut être utile de signaler que les questions sur le sens objectif des faits sociaux sont redevenues très actuelles à la suite d'une série de travaux récents en philosophie des sciences sociales qui ont essayé d'intégrer l'apport des sciences cognitives contemporaines pour préciser le statut ontologique des faits sociaux ${ }^{10}$. Ainsi par exemple, le philosophe J. Searle a-t-il développé il y a peu une hypothèse sur «la construction de la réalité sociale » qui, partant également du constat du caractère en partie immatériel des faits sociaux, qu'il appelle aussi faits institutionnels, propose de les traiter comme des faits bruts auxquels les hommes auraient attribué conventionnellement une certaine valeur ou fonction dans certains contextes ${ }^{11}$. Toutefois, Searle suppose aussi que cette attribution de valeurs est le fait d'une intentionnalité collective qui repose elle-même sur des mécanismes psychologiques individuels dont l'explication ultime est à trouver dans les sciences naturelles et, en fait, dans le fonctionnement du cerveau. Autrement dit, il emprunte la voie réductionniste que Durkheim avait voulu éviter, en particulier lorsqu'il se préoccupait de constituer la sociologie comme science indépendante de la biologie et du darwinisme social ${ }^{12}$. D'autre part, Searle n'évite pas non plus les écueils classique du conventionnalisme qui, lorsqu'il est utilisé à des fins d'explication fondamentale, pose inévitablement un problème de régression logique, car on a toujours besoin d'une convention antérieure pour expliquer une convention quelconque.

A l'opposé de cette démarche psychologisante, Vincent Descombes a défendu dans un autre ouvrage récent ${ }^{13}$ une hypothèse holiste, inspirée de Marcel Mauss et

8 Cf., dans cette direction, toute l'œuvre de R. Boudon qui met en évidence les vertus explicatives du traitement des raisons comme des causes de l'action sociale. Cf. par exemple R. Boudon, L'art de se persuader, Paris, Fayard, 1990.

9 Cf. A. Schutz, Der sinnhafte Aufbau der sozialen Welt, eine Einleitung in die verstehende Soziologie, Frankfurt, Suhrkam, tr. ang. The Phenomenology of the Social World, Evanston, Northwestern University Press, 1967, éd. orig. 1932.

10 Cf. A. Bouvier A., P. Livet éd., L'ontologie des faits sociaux, in « Raisons pratiques », 2000.

11 J. Searle, La construction de la réalité sociale, tr. fr. C. Tiercelin, Paris, Gallimard, 1998, éd. orig. 1995 , p. 46 et sq.

12 Cf., à ce sujet, l’ouvrage bien documenté de J.M. Bernardini, Le darwinisme social en France, Fascination et rejet d' une idéologie, Paris, CNRS Editions, 1997.

13 Les institutions du sens, Paris, Minuit, 1996. 
de Louis Dumont, qui propose de traiter les institutions comme un ensemble de relations d'ordre qui seraient l'émanation de la vie en commun. Descombes introduit, à cette occasion, une notion d' «esprit objectif» qu'il trouve chez MerleauPonty (ibid., p. 282) et qui est une façon de formuler le problème d'objectivité posé par le caractère intrinsèquement significatif des faits sociaux. Cet esprit objectif que Descombes considère comme un esprit essentiellement pratique (ibid., p. 28), pourrait faire penser aux thèses pragmatistes de G. Mead sur les institutions comme réponses communes des membres d'une communauté à une situation particulière, fondées sur l'attitude de l' autre généralisé » ${ }^{14}$. Mais Descombes évoque surtout Hegel et l'«antériorité des droits de l'esprit objectif sur ceux de l'esprit subjectif ${ }^{15} »$, et, s'inspirant de Wittgenstein, il insiste in fine sur « la priorité d'une règle sur l'activité qu'elle gouverne»(ibid., p. 330). La notion hégélienne d'esprit objectif repose toutefois sur un rapprochement délicat entre deux notions apparemment peu compatibles, celles d'esprit et d'objectivité. Un tel rapprochement ne pourrait tenir que si l'on disait en quoi l'esprit, qu'on envisage habituellement comme quelque chose de plutôt « subjectif », car il ne se révèle que dans une expérience psychologique interne, peut aussi être objectif. Or, Descombes, qui reprend à son compte les thèses de Quine sur l'indétermination des significations, rejette l'idée de significations objectives (ibid., p. 330), de sorte qu'on ne voit plus très bien ce qu'il pourrait y avoir à objectiver dans les règles sociales, en-dehors des régularités de comportement qu'elles induisent. Le problème serait de dire, non pas seulement s'il y a quelque chose comme un esprit objectif qui se manifeste dans des règles sociales, ce qui n'est qu'une autre façon d'énoncer le problème durkheimien initial, mais en quoi consiste précisément l'objectivité du contenu des règles sociales dont découle leur pouvoir social contraignant.

Les difficultés soulevées par la démarche de Descombes ne sont du reste pas très éloignées de celles de la tradition sociologique issue de l'interactionnisme symbolique et de la phénoménologie sociale qui considère que la réalité des faits sociaux repose sur des significations, mais aussi que ces faits sont socialement construits $^{16}$, ce qui veut dire que leurs significations sont le résultat d'attributions de sens opérées par les acteurs lorsqu'ils ont à se désigner eux-mêmes, à désigner leurs actions ou les objets qui les entourent - ces attributions de sens pouvant elles-mêmes être régulées par la culture ${ }^{17}$ ou les actions conjointes ${ }^{18}$. Cette ligne de pensée, dont sont issus différents courants en sociologie des sciences ou en sociologie de la déviance ${ }^{19}$, néglige cependant le fait important que la désignation des « choses », qu'il s'agisse d'un objet de la science physique ou d'une action cri-

14 Cf. G.H. Mead, Mind, Self and Society, Chicago, University of Chicago Press, 1962, éd. orig. 1934, vol. 1, p. 261.

15 Les institutions du sens, op. cit., p. 289.

16 Suivant le titre du fameux livre de P. Berger et T. Luckmann, La construction sociale de la réalité, tr. fr. P. Taminiaux, Paris, Méridiens Klincksieck, 1987, éd. orig. 1966.

17 Cf. H. Becker, Outsiders, tr. fr. J.P. Briand et J.M. Chapoulie, Paris, A.M. Métailié, 1985, éd. orig., 1963.

18 Cf. H. Garfinkel, Studies in Ethnomethodology, Cambridge, Polity Press, 1984, éd. orig. 1967.

19 Cf. A. Ogien, Sociologie de la déviance, Paris, Nathan, 1998. 
minelle, est généralement soumise à des régulations objectives qui s'expriment dans les termes sémantiques du vrai et du faux et rendent en principe impossible la confusion entre des choses incompatibles, comme par exemple un viol et une caresse amicale, un pulsar et un tas de cailloux ou un professeur et un écolier. Même s'il est vrai que toutes sortes de règles sociales peuvent être «construites » par les agents pour se désigner entre eux ou pour désigner leurs actions ou leur environnement, que ces règles sont donc « relatives » à certains contextes ou à certaines cultures, il ne semble pas vrai que ces règles ne soient soumises à aucune contrainte d'objectivité plus générale et non-relative à ces contextes. Tout le problème de la tradition interactionniste ou phénoménologique en sociologie est précisément qu'elle laisse béante cette question du contenu objectif des règles de signification.

Ce bref parcours permet finalement d'apercevoir la difficulté principale que l'on rencontre lorsqu'on s'interroge sur le sens objectif des faits sociaux et qui tient au caractère abstrait et immatériel de «choses» logico-sémantiques telles que les pensées, les significations, les motifs, les valeurs, les idéologies, dont l'objectivation ne peut pas se faire de la même façon que celle des déplacements des corps. Dans les faits, la plupart des données sociologiques empiriques, qu'elles soient ethnographiques ou statistiques, se rapportent aux deux sortes de «choses » qu'on peut repérer dans la vie sociale: déplacements de corps ou contenus logicosémantiques. Mais comme la tendance matérialiste générale de la science moderne recommande d'éviter les facilités du platonisme, qui admet l'existence d'entités idéales et invisibles, ou du dualisme cartésien qui sépare le monde en deux sortes d'entités, étendues ou pensantes, on peut être tenté, pour faire face à la difficulté, soit de ramener les pensées et les significations à des réalités biologiques sous-jacentes, ce qui conduit à un réductionnisme naturaliste (cf. infra, $3^{\mathrm{e}}$ section), soit d'adopter une position relativiste qui refuse d'accorder un quelconque statut d'objectivité aux significations attribuées. Il me semble que la meilleure façon d'éviter les inconvénients de ces tentations réductionnistes ou relativistes serait d'admettre que la société humaine, comme semble d'ailleurs l'avoir supposé Durkheim, est une «chose» à la fois pensée et pensante dont il faut pouvoir rendre compte pour ce qu'elle est, ce qui implique de se donner les outils méthodologiques adéquats. Weber avait clairement admis ce point, mais d'autres sociologues après lui en ont également tenu compte, en particulier Parsons. C'est donc vers eux que je vais maintenant me tourner.

\section{SOCIOLOGIE COMPRÉHENSIVE ET RÉALISME ANALYTIQUE ${ }^{20}$}

A la différence de Durkheim, Weber ne parle pas de faits sociaux mais plutôt d'événements, de phénomènes et, plus généralement, d'ensembles significatifs (Sinnzusammenhang) ouverts à la compréhension. Et tandis que Durkheim voyait la spécificité des faits sociaux dans des règles normatives extérieures aux individus, Weber la voit plutôt dans le sens que les agents confèrent, de l'intérieur, à

20 Je remercie Thierry Blin d'avoir attiré mon attention sur l'importance de cette notion chez Parsons. 
leurs actions. On a évidemment beaucoup glosé sur cette différence de point de vue entre les deux auteurs. Mais au fond, je ne suis pas sûr qu'elle soit aussi radicale, car en fait les deux analyses rencontrent à peu près le même genre de difficultés. Le problème de Weber, en effet, est aussi de spécifier un domaine social qui ne soit ni celui des réactions biochimiques, ni celui d'une vie psychique conçue comme organisation d'éléments isolés indépendamment de leur sens communicable ${ }^{21}$. Weber se préoccupe en particulier de distinguer les faits dépourvus de sens, qui peuvent être physiques, astronomiques, géologiques..., mais aussi psychopathologiques (ibid., p. 12), des faits auxquels les agents attribuent un sens. Or, pour lui, le sens ou la signification n'est pas du tout un phénomène psychologique, mais plutôt un effet de présuppositions rationnelles (ibid., p. 17). Le modèle interprétatif fondamental de Weber est en effet celui de la compréhension d'une opération arithmétique (ibid., pp. 5, 17) qui ne passe pas du tout par la psychologie mais s'appuie au contraire sur des règles rationnelles extrapsychologiques $^{22}$. Weber étend ce modèle à toutes les présuppositions rationnelles qu'on applique à l'action et qui permettent à la fois de la comprendre dans son sens visé, et éventuellement de l'expliquer causalement lorsqu'il y a correspondance entre le déroulement extérieur de l'activité et le sens ou les motifs qu'on lui reconnaît. C'est d'ailleurs pour cette raison que Weber se montre aussi circonspect sur la possibilité de comprendre un comportement irrationnel.

L'approche wébérienne du phénomène social est, d'une certaine façon, plus englobante que celle de Durkheim puisqu'elle inclut, sous la notion d'ensemble significatif, soit un événement particulier, qui n'est pas forcément individuel, soit un comportement collectif moyen qui peut être saisi par une statistique, soit enfin un type pur construit à partir d'un phénomène ayant une certaine fréquence (ibid., p. 6). Weber propose en outre un critère précis de l'ensemble significatif par opposition aux faits objectifs qui ne sont pas compréhensibles au sens où il l'entend. Ce critère est celui du lien entre le phénomène et le contenu significatif visé par l'action humaine à laquelle il se rapporte, soit comme moyen, soit comme fin. Par exemple, un événement particulier tel qu'une guerre ou une rencontre amoureuse pourrait être rapportée à un sens de l'action, tandis que ce n'est pas du tout le cas d'une division cellulaire. De même, on pourrait, selon Weber, appliquer son critère aux statistiques de la criminalité, de la profession, des prix ou des exploitations agricoles, mais pas à la mortalité, la fatigue, le rendement d'une machine ou les chutes de pluie (ibid., p. 11). Quant aux types purs, il y en a certains comme l'éthique protestante qui ne s'appliquent à l'évidence qu'aux phénomènes qui relèvent d'une action sensée.

On peut évidemment adresser pas mal d'objections au modèle wébérien du phénomène social. On dira par exemple qu'il existe des faits naturels dépourvus de sens visé qui sont aussi des faits sociaux, comme par exemple le drame du Heysel causé par un mur qui s'effondre sur les spectateurs paniqués d'un match de football ou bien la sénilité de Boris Eltsine qui menaçait, à un certain moment, l'équilibre international, ou encore le ralentissement de la croissance démographique mondiale. Mais Weber tient compte de ce genre de cas en les traitant

21 Economie et société, tr. fr. J. Freund et al., Paris, Plon, 1971, éd. orig. 1921, p. 21.

22 Ce qui rapproche sa vision du sens de celle de logiciens tels que Frege (cf. infra). 
comme des occasions de l'activité significative des agents humains (ibid., p. 7). Le phénomène naturel, selon lui, n'a pas de sens interne, et ne devient social que du point de vue de son insertion dans des enchaînements qui, eux, ont un sens interne. Une autre objection serait que les animaux peuvent aussi développer une activité significative, ce dont Weber ne disconvient pas (ibid., p. 14), mais il souligne que ce sont les analogies humaines, et non l'inverse, qui permettent de prêter un sens visé aux activités animales (ibid., p. 15). Et Weber ajoute qu'on ne pourrait réduire les phénomènes sociaux à des faits naturels qu'à la condition d'avoir préalablement découvert des critères expérimentaux de l'orientation significative (ibid., p. 15), ce dont on est encore loin, y compris dans les sciences éthologiques contemporaines ${ }^{23}$.

On pourrait encore objecter que les objets sociaux tels que les institutions, les structures ou les courants sociaux dont parlait Durkheim, cadrent mal avec l'analyse wébérienne, qui est beaucoup trop individualisante. Mais, sur ce sujet aussi, on peut trouver des éléments de réponse chez Weber lorsqu'il dit que ces structures sociales « sont des représentations de quelque chose qui est, pour une part, de l'étant, pour une autre part, du devant-être, qui flotte dans la tête des hommes réels (...) d'après quoi ils orientent leur activité ${ }^{24} »$. C'est ici toutefois que l'analyse wébérienne peut paraître dramatiquement incomplète. On aimerait en effet savoir plus précisément comment des significations ou des pensées, qui ne sont pas des choses à proprement parler, sont susceptibles de s'incarner dans des choses réelles comme des actions ou des objets sociaux et d'avoir certains effets concrets dans la réalité sociale. Weber considérait peut-être cette question résolue par le montage épistémologique et méthodologique élaboré pour analyser l'action à partir d'une notion de sens qui, par définition, ne peut inclure des êtres physiques. Mais, ce faisant, il a laissé non résolu et ouvert à toutes les conjectures le problème du statut de ces choses mi-réelles et mi-virtuelles qui «flottent dans la tête des hommes réels » et déterminent le contenu de leurs actes et de leurs pensées.

C'est à ce point qu'on touche l'objection la plus radicale que l'on pourrait adresser à la thèse wébérienne. Il s'agit d'une objection de type logique qui consiste simplement à dire que le sens visé n'est pas un particulier susceptible d'entrer dans le parcours de valeur d'une variable propositionnelle, et qu'il n'est donc pas susceptible d'être décrit suivant les moyens habituels de la science positive. Il semble d'ailleurs que toutes les attaques contre la sociologie compréhensive soient fondées, explicitement ou implicitement, sur cette objection, et sur son arrière-plan de logique extensionnelle et d'épistémologie physicaliste, largement dominantes dans la philosophie des sciences contemporaines. Une justification fondamentale de cette critique pourrait être trouvée dans l'opposition classique que G. Frege ${ }^{25}$ avait établie entre le sens (Sinn), qui fixe le sens ou la valeur cognitive d'une proposition - par exemple «l'étoile du matin»-, et sa dénotation

23 Cf. sur ces sujets l'intéressant dossier de «Pour la science », L'intelligence, $\mathrm{n}^{\circ}$ M 2687, déc. 1998, et, sur l'intelligence animale, l'ouvrage de D. Desor, Le comportement social des animaux, Presses Universitaires de Grenoble, 1999.

24 Economie et société, op. cit., p. 13.

25 Sens et dénotation, tr. fr. C. Imbert, in Ecrits logiques et philosophiques, Paris, Seuil, 1971, éd. orig. 1892. 
(Bedeutung), qui fixe habituellement sa valeur de vérité - dans l'exemple, il s'agit de l'objet désigné par «étoile du matin». Or, selon Frege, il existe un seul cas où le sens d'une proposition peut devenir la dénotation d'une autre proposition, c'est celui du style indirect, du type par exemple: «A croit que $\mathrm{p}$ ». Mais ce cas est aussi le seul où la dénotation de la proposition (ce que croit A) n'est pas aussi une valeur de vérité, car un sens ou une croyance ne peut pas être un objet (ibid., p. 105).

On peut facilement appliquer cette objection logique à toute prétention de description objective des pensées, des valeurs ou des significations sociales qui, n'étant pas des objets extensionnels identifiables, ne peuvent pas non plus relever d'une description objective, conforme par exemple au modèle canonique des descriptions russelliennes: « il existe un x tel que... », dans le cadre de la logique des prédicats du premier ordre. Sur le plan méthodologique, l'objection se traduit par le fait qu'il n'existe en effet aucun moyen expérimental de s'assurer qu'un individu a bien la pensée qu'on lui prête ou qu'une institution a bien la signification qu'on lui attribue: par exemple une même action peut exprimer plusieurs sens différents, ou bien une simple observation externe peut donner lieu à des contresens, ou encore une interrogation de l'agent sur le sens de son action peut entraîner des réponses inexactes, soit que l'agent ne veuille pas dire ce qu'il pensait en agissant, soit qu'il soit incapable de formuler cette pensée, soit parce qu'il ne le sait pas luimême, etc. ${ }^{26}$ Ces difficultés bien connues ont été à la base du rejet de la sociologie compréhensive par différents courants fonctionnalistes ou opérationnalistes de la sociologie. Mais comme aucune démarche sociologique n'a jamais réussi à analyser un phénomène social en faisant complètement abstraction de son sens endogène, il est également impossible de se débarrasser de la sociologie compréhensive.

Certains critiques de Weber, tels que Parsons, ont aperçu la difficulté mais ne l'ont pas pour autant résolu. Parsons reproche en particulier à l'approche idéaltypique de Weber un «fictionnalisme» qui réduirait la réalité sociale à une construction idéale $^{27}$. Mais Parsons n'est peut-être pas tout à fait juste à l'égard de Weber, car si celui-ci était certainement moins réaliste que Durkheim, son éventuel «nominalisme»n'a jamais été jusqu'à mettre en cause la réalité objective du phénomène social. Weber défend d'ailleurs, dans ses Essais sur la théorie de la science, une théorie de la sélection descriptive des fragments de réalité qui semble tout à fait compatible avec des formes ultérieures de réalisme scientifique, y compris celui de Parsons ${ }^{28}$. D'un autre côté, lorsque Parsons critique le chosisme ou le réalisme social de Durkheim et sa tendance à traiter la réalité sociale comme une entité métaphysique, il soutient à la fois que les faits observables sont toujours des faits matériels qui ne sont pas à proprement parler des faits sociaux, ce qui semble indiscutable, mais aussi, suivant une inspiration très wébérienne, qu'il n'existe en fait que des esprits individuels, et pas d'esprit collectif ${ }^{29}$. Mais cette critique laisse

26 Toutes ces difficultés avaient déjà été répertoriées par M. Weber dans les premières pages d'Economie et société.

27 The Structure of Social Action, rééd. 1968, 2 vol., New York, Free Press, 1937, p. 730.

28 Cf. par exemple ses développements dans L'objectivité de la connaissance dans les sciences et la politique sociales, tr. fr. J. Freund et al., Essais sur la théorie de la science, Paris, Plon, 1965, éd. orig. 1904, pp. 152 et sq.

29 The Structure of Social Action, op. cit., p. 358. 
intacte l'objection logique énoncée précédemment, laquelle porte sur le statut ontologique du sens, qu'il soit exprimé individuellement ou qu'il le soit collectivement.

Parsons, il est vrai, ne tire aucune conclusion béhavioriste ou physicaliste de sa critique du fictionnalisme wébérien et du réalisme durkheimien, puisqu'il rejette aussi l'empirisme brut ${ }^{30}$ et pense que l'erreur majeure serait de traiter la réalité sociale comme un objet séparé spatialement ${ }^{31}$, alors qu'il ne s'agit que d'une abstraction théorique. Pour lui, la réalité sociale n'est pas une classe de choses concrètes indépendantes, mais un ensemble d'éléments dans un système d'action total qui comprend aussi l'hérédité, l'environnement, les désirs (ibid., p. 368). Parsons peut ainsi opposer aux différentes sortes d'empirisme relatif à l'action (ibid., p. 728) un «réalisme analytique» qui consiste à traiter ce qu'il considère comme les éléments de base de l'action, - qui sont pour lui les conditions, les moyens, les fins et les normes de l'action -, comme des propriétés émergentes à un certain niveau de complexité du réel (ibid., p. 741). Comme chez Weber, le problème essentiel de Parsons dans le dernier chapitre de La structure de l'action sociale est de relier la réalité purement logique de l'action - il dit par exemple que « du seul point de vue objectif, toute action est «logique»» (ibid., p. 733) - et sa réalité concrète, c'est-à-dire spatio-temporelle, accessible aux sens et aux autres sciences. Pour cela, l'idée principale, exprimée notamment par une métaphore graphique du tissage de la réalité par les constituants de l'action (ibid., p. 741), est qu'on peut procéder à différentes séparations analytiques sur des parties concrètes du réel qui, en réalité, ne sont pas vraiment séparées. L'unité d'action (action unit) est un cas de séparation analytique parmi d'autres, comme par exemple les séparations analytiques opérées par la physique (ibid., p. 737). Quand on parle d'action, on parle donc bien, selon Parsons, de quelque chose de réel, au sens concret du terme, mais qu'on traite uniquement du point de vue des constituants analytiques de l'action. La difficulté, pour la science sociale, est donc de situer la structure proprement agentive des phénomènes réels par rapport à ce qui relève en eux de la seule causalité physique. Parsons, qui cite Husserl à plusieurs reprises (ibid., p. 733 et 750), semble chercher ici une solution du problème en terme d'autonomie phénoménologique du cadre de référence de l'action susceptible de se transformer en «processus subjectifs réels» (ibid., p. 750).

Dans toute cette démarche, Parsons reste fidèle à l'idée classique de l'action, comme mouvement spontané, intentionnel et réfléchi, par opposition à un comportement physique naturel. L'action, avec ses propriétés particulières, est en fin de compte une sorte de réalité émergente qui doit donner lieu à des descriptions distinctes des descriptions physiques, et la modélisation qu'en propose Parsons anticipe d'une certaine façon sur des travaux ultérieurs en philosophie de l'action $^{32}$. Cependant, malgré son raffinement et sa puissance, ce modèle épistémologique ne parvient pas à surmonter l'objection logique énoncée précédemment.

The Social System, London, Tavistock Publications Ltd. 1951, p. 20.

The Structure of Social Action, op. cit., p. 367.

32 Cf. D. Davidson, Actions et événements, tr. fr. P. Engel, Paris, PUF, 1993, éd. orig. 1980, et le compte-rendu que j'en ai fait, dans « l'Année Sociologique », vol. 46, 1995, pp. 434-440. 
Ainsi, dans un article de $1938^{33}$, Parsons a proposé de traiter les idées - prises au sens des concepts et propositions empiriques, non-empiriques ou normatives intelligibles par les acteurs -, comme des variables indépendantes susceptibles de faire partie de plein droit d'un schéma empirique d'explication. L'exemple du rôle des idées calvinistes dans le développement économique sert de paradigme à l'analyse, puisque, selon Weber, lorsque cette variable est absente, la développement économique n'a pas lieu. Mais l'objection logique, reformulée par exemple dans les termes de la critique wittgensteinienne, serait ici qu'on ne peut pas séparer les idées calvinistes de la pratique dans laquelle elles s'incarnent, car la description des idées d'une pratique n'est qu'une autre façon de décrire la même pratique. Autrement dit, il n'existe aucun être singulier, aucun objet d'expérience tel que des pensées calvinistes qui pourrait être la valeur d'une variable indépendante relative aux types de pensées. Si cette objection était justifiée, la mention des idées calvinistes ne serait au fond qu'une redondance sur la description de la pratique calviniste, et pas du tout une explication. Dans le même ordre d'idée, il serait illusoire de vouloir expliquer des phénomènes historiques par les idéologies des agents, comme par exemple le nazisme par l'antisémitisme allemand ${ }^{34}$.

On touche ici à un problème qui va devenir central avec le développement des sciences cognitives contemporaines, celui de l'indépendance logique des contenus cognitifs par rapport aux déterminants écologiques et physiologiques de l'action humaine. L'analyse parsonienne, comme du reste celle de Weber, suppose, à juste titre me semble-t-il, l'indépendance des contenus sémantiques: idéologies, valeurs, motifs, par rapport au déclenchement physique de l'action et aux effets qu'elle a sur autrui, mais elle n'en propose aucune démonstration. Le niveau systémique-fonctionnel auquel Parsons se situe dans la suite de son œuvre, et qui l'éloigne de plus en plus de la sociologie compréhensive ${ }^{35}$, semble d'ailleurs le dispenser d'apporter ce type de précisions en lui permettant de négliger la composante du sens subjectif de l'action, comme cela lui fut souvent reproché ${ }^{36}$. Mais, par la même occasion, Parsons échoue à clarifier la place du système d'action à l'intérieur des phénomènes naturels. Et de fait, si on admet le réalisme analytique de Parsons en ce qui concerne l'action, on est sans doute protégé contre l'élimination pure et simple de la catégorie de l'action douée de sens comme vecteur principal du fait social, mais pas suffisamment contre une réduction de ses différentes composantes à des phénomènes organiques plus basiques, ni contre le scepticisme sur la possibilité d'isoler dans la réalité une causalité propre de l'action par rapport aux causes naturelles.

33 The Role of Ideas in Social Action, in Essays in Sociological Theory, London, The Free Press, 1949, éd. orig. 1938.

34 Cf. sur ce sujet les efforts de D. J. Goldhagen, Les bourreaux volontaires de Hitler, les Allemands ordinaires et l'holocauste, Paris, Seuil, 1997, éd. orig. 1996, pour s'opposer aux explications interactionnistes et pragmatistes du nazisme qui minorent le rôle des idées antisémites.

35 Cf. son commentaire sur ce point dans une préface tardive (1968) de The Structure of Social Action, op. cit, p. X-XI.

36 Cf. en particulier F. Isambert, Les avatars du fait moral, in De la religion à l'éthique, Paris, Cerf, 1992, éd. orig. 1982. 


\section{SÉMANTIQUE DES FAITS SOCIAUX}

Pour mieux apercevoir la portée de l'objection logique relative à l'identification et à l'objectivation du sens et des pensées comme « variables indépendantes ", il peut être utile d'en mesurer certaines conséquences méthodologiques. Si, comme le pensent beaucoup d'auteurs, cette objection est fondée, les sciences sociales seraient confrontées à un dilemme qui les obligerait soit à renoncer à une entreprise de description et d'explication scientifique du sens des faits sociaux, en considérant par exemple que, sur ce plan, leur tâche est essentiellement de nature interprétative, ce qui est une position assez courante, au-delà même du cercle étroit de la sociologie dite interprétative, soit à trouver au sens et aux pensées un substrat objectif plus facilement identifiable. Pour cela, les sciences sociales devraient essayer soit d'induire le sens et les pensées à partir de faits comportementaux plus basiques, comme par exemple des structures et mouvements de population, des formes d'organisation ou de peuplement, des phénomènes de différenciation ou de stratification des sociétés, etc., ce que fait du reste déjà la statistique sociale, sans toutefois prétendre disposer d'une véritable méthode d'induction du sens des phénomènes; soit, de façon plus radicale, de les réduire à des mécanismes comportementaux ou neurophysiologiques, qui, eux, seraient en mesure de fixer la valeur des variables cognitives d'un discours explicatif, dans la mesure où il seraient eux-mêmes déjà des mécanismes cognitifs. C'est précisément à ce point que peut être envisagée une entreprise de naturalisation des faits sociaux qui, à la différence de l'ancien darwinisme social, privilégie l'approche cognitive, comme le font aujourd'hui la sociobiologie ${ }^{37}$ mais aussi l'anthropologie ou la psychologie évolutionnaire ${ }^{38}$.

La naturalisation des faits sociaux peut ainsi s'inscrire dans une entreprise générale de naturalisation des fonctions cognitives et morales sur la base de schémas analytiques directement inspirés des sciences du vivant. Par rapport à la tradition béhavioriste qui avait parfois tendance à nier purement et simplement le rôle des pensées et des faits cognitifs dans les comportements humains ${ }^{39}$, l'apport des sciences cognitives contemporaines a consisté au contraire à proposer une explication de cette classe de faits, fondée sur la neurophysiologie et l'évolution naturelle. Pendant un moment, sous l'effet du succès du computationalisme et de sa représentation du cerveau sur le modèle d'un calculateur électronique, la naturalisation de l'esprit a surtout été conçue sur le modèle de systèmes symboliques physiques produisant des représentations mentales ${ }^{40}$. Ces modèles, et en particulier les modèles connexionistes, qui rendent, semble-t-il, de grands services à l'étude des fonctionnements neurocérébraux ${ }^{41}$, sont loin d'être abandonnés

37 Cf. J. Lopréato, Le défi de la sociobiologie à la science de la culture, in « Revue Européenne des sciences sociales », n 69, vol. XXIII, Genève, 1985, pp. 21-67.

38 Cf. D. Sperber, La contagion des idées, Paris, Odile Jacob, 1996, J. H. Barkow, L. Cosmides L., Tooby J. eds., The Adapted Mind, Evolutionary Psychology and the Generation of Culture, Oxford, Oxford University Press, 1992.

39 Cf. B.F. Skinner, Verbal Behavior, New York, Appleton-Century-Crofts, 1957.

40 Cf. A. Newell, Physical Symbol Systems, in D.A. Norman ed., Perspectives on Cognitive Science, Ablex, Norwood, 1981, pp. 37-86.

41 Cf. par exemple J.-P. Nadal, L'intelligence des réseaux de neurones, in « Pour la science », $\mathrm{n}^{\circ}$ M 2687, 1998, pp. 164-172. 
aujourd'hui par les sciences cognitives, mais les théoriciens cherchent aussi à les intégrer à des mécanismes de stimulation externe qui commanderaient le fonctionnement interne des organismes. Dans cette perspective, on fait appel à deux types de principes:

- un principe de causalité naturelle qui explique la formation des croyances par les effets que les informations du monde extérieur exercent sur l'appareil cognitif des êtres vivants, celui-ci étant conçu comme une structure physique capable d'effectuer des opérations symboliques, et en particulier des calculs ${ }^{42}$;

- un principe d'économie naturelle qui pousse les organismes à traiter en priorité les informations qui leur sont le plus profitables par l'élimination des organismes qui ne respectent pas ce principe - le fait de la survivance étant aussi le critère ultime de ce qui est profitable ou utile à un organisme ${ }^{43}$.

Il ne saurait être question de dresser ici un tableau des multiples aspects de cette entreprise générale de naturalisation de l'esprit, y compris de la logique, de la morale, de l'économie et des faits sociaux, dans le cadre des sciences cognitives contemporaines. Mais il me semble important de souligner qu'un tel programme se heurte inévitablement au problème du statut ontologique du sens et des pensées, dont l'une des caractéristiques les plus habituellement reconnues est de ne pas avoir de réalité sensible. Comme l'avait déjà remarqué Frege, les pensées ne sont certainement pas des choses du monde extérieur, car elles ne peuvent pas être touchées, ni prises dans les mains, ni observées et elles n'apparaissent de façon sensible que sous la forme voilée du langage et de nulle autre façon ${ }^{44}$. Mais les pensées ne sont pas non plus des représentations psychologiques car, bien qu'elles ne soient ni sensibles ni accessibles par un autre moyen que la conscience interne, elles peuvent être reconnues à l'identique par plusieurs penseurs ${ }^{45}$ et leur vérité est indépendante du fait qu'elles sont pensées par un penseur particulier. Autrement dit, l'objectivité des pensées est à la fois logique et sociale ${ }^{46}$, et sa meilleure illustration est celle des pensées mathématiques qui demeurent inchangées lorsqu'elles se transmettent de sujet à sujet. Frege remarquait en outre que, bien qu'elles ne soient pas des êtres sensibles, les pensées ne sont pas causalement inertes car elles agissent sur les hommes par le fait qu'elles sont «saisie(s) et

42 Cf. en particulier J. Fodor, A., Theory of Content and Other Essays, Cambridge, MIT Press, 1991 et P. Jacob, Pourquoi les choses ont-elles un sens?, Paris, Odile Jacob, 1997.

43 Cf. F. Dretske, Explaining Behavior, Cambridge, MIT Press, 1988, D. Dennett, La stratégie de l'interprète, tr. Fr. P. Engel, Paris, Gallimard, 1990, éd. orig. 1987, R. G. Millikan, Biosemantics, in « The Journal of Philosophy », vol. LXXXVI, n 6, juin 1989, pp. 281-297.

44 G. Frege, Recherches logiques, 1, La pensée, tr. fr. C. Imbert, in Ecrits logiques et philosophiques, Paris, Seuil, 1971, éd. orig. 1918-1919, p. 180. La notion frégéenne de pensée (Gedanke) désigne le sens des propositions, susceptibles d'être vraies ou fausses si elles sont complètes, c'est-à-dire si elles incluent certaines spécifications de temps et de lieu. Les termes singuliers ont également un sens, mais ces sens ne deviennent des pensées que lorsqu'ils sont inclus dans des propositions complètes.

45 Frege prend l'exemple du théorème de Pythagore (ibid., p. 183) et il parle d'un «trésor commun des pensées qui se transmet d'une génération à l'autre», Sens et dénotation, op. cit., p. 106.

46 Recherches logiques, I, La pensée, op. cit., p. 199. 
tenue(s) pour vraie(s)» (ibid., p. 194). Enfin, nos actes sont ordinairement préparés par l'acte de penser et de juger et «l'action de l'homme sur l'homme est la plupart du temps médiée par des pensées ${ }^{47} \gg$.

Ces brèves indications permettent peut-être de sortir du dilemme exprimé au début de cette section, avec son prolongement dans un programme comportementaliste de naturalisation des faits cognitifs et sociaux. En effet, pour faire face à ce dilemme, la meilleure stratégie n'est sans doute pas de se résigner aux approximations d'un travail interprétatif mal défini, non seulement parce que l'interprétation est souvent une pratique vague et peu satisfaisante, mais aussi parce que ce repli ne rendrait pas compte du travail de la sociologie tel qu'il se fait réellement, lequel a un caractère effectivement objectif et descriptif, même lorsqu'il porte sur du sens, des pensées, des motifs, des valeurs. Personne ne doute par exemple que les idées protestantes ou les idées antisémites sont logiquement distinctes, respectivement des idées hindouistes ou cosmopolites. Le problème est seulement qu'on ne sait pas exactement quel est le statut ontologique et épistémologique de cette différence par rapport aux faits directement observables, d'où la question parsonienne sur le statut des idées comme variables indépendantes. Ce problème était du reste déjà celui des marxistes qui l'avaient résolu, eux, à l'inverse de Parsons, en supposant que les superstructures idéologiques d'une société ne sont qu'une sorte de déformation, on disait aussi de «reflet», de ses infrastructures qui seules peuvent donner le sens objectif des faits sociaux - la difficulté étant ici qu'on voit mal comment des phénomènes qui n'ont pas déjà un sens, tels que les processus matériels du développement productif, pourraient eux-mêmes donner le sens des faits historiques.

Mais la bonne stratégie ne consiste pas non plus, me semble-t-il, à dédaigner l'objection logique en faisant mine de considérer le sens et les pensées comme des particuliers existants, observables et de bon aloi, qui fixeraient directement la valeur de nos variables propositionnelles sur l'action et les faits sociaux en général. La raison n'est pas seulement que cette stratégie s'oppose aux règles canoniques d'une logique extensionnelle largement admise aujourd'hui en philosophie des sciences, car on pourrait dire qu'après tout cette logique n'est pas la seule possible $^{48}$ ou bien que les outils de la logique peuvent être séparés des questions d'existence ${ }^{49}$ ou encore que, même dans le cadre d'une logique extensionnelle, il est possible de trouver un traitement aux variables intensionnelles en les traitant comme des types de propriétés ${ }^{50}$. C'est surtout que cette indifférence peut priver

47 Recherches logiques, 2, La négation, tr. fr. C. Imbert, in Ecrits logiques et philosophiques, Paris, Seuil, 1971, éd. orig. 1918-1919, p. 199.

48 Il existe aussi en effet des logique intensionnelles, et non pas extensionnelles. Cf. par exemple R. Montagut, Formal Philosophy, New Haven, Yale University Press, 1974.

49 Il est possible par exemple, contrairement à ce qu'envisageait Quine suivant son fameux principe d'engagement ontologique, de séparer la quantification logique de toute question d'existence.

50 Il existe dans la littérature logique contemporaine un grand nombre de stratégies pour rendre le sens et les pensées compatibles avec la forme canonique des descriptions russelliennes. La plus célèbre est celle de D. Davidson (La forme logique des phrases d'action, tr. fr. P. Engel, in Actions et Evénements, Paris, PUF, 1993, éd. orig. 1967) au sujet de la forme logique des phrases d'action qui consiste à lier la variable d'action d'une proposition à un événement indéterminé auquel sont attribuées certaines propriétés intentionnelles. Par exemple «A se marie avec B » s'énonce comme suit: « il existe un événement $\mathrm{X}$ tel que $\mathrm{X}$ est le mariage de $\mathrm{A}$ et de $\mathrm{B}$ ». Suivant ce pro- 
le sociologue des outils méthodologiques nécessaires à son travail d'objectivation du sens et des pensées en lui faisant privilégier d'illusoires outils observationnels au détriment d'outils logiques et conceptuels qui seuls permettent d'étudier réellement le sens et les pensées.

Il suffit en effet de réfléchir un instant pour voir que s'il est possible de séparer logiquement des pensées, il est en revanche impossible de les séparer par l'observation empirique car, comme le montre la diversité des langues et des systèmes de signe, des pensées identiques peuvent se manifester sous des formes perceptuelles distinctes, et, à l'inverse, on ne peut jamais exclure que des pensées différentes se manifestent sous des formes perceptuelles identiques. Pour reprendre ici, d'une façon un peu détournée, un argument quinien sur l'indétermination de la traduction $^{51}$, on ne peut jamais exclure que les mêmes mouvements physiques, et en particulier ceux qui sont constitutifs des actes sociaux, puissent donner lieu, en termes de pensées exprimées par l'agent, à deux traductions radicalement différentes et cependant compatibles avec les mêmes données sensibles. Mais contrairement à la conclusion qui était celle de Quine, cet argument n'exclut pas que les pensées possèdent une certaine objectivité mais montre seulement que cette objectivité n'est pas de l'ordre de ce qui s'observe dans la perception sensible, puisqu'il n'existe pas de capteurs physiques des pensées et que cependant nous sommes capables de les différencier.

Le meilleur moyen d'expliquer la robustesse des explications sociologiques par des idées est sans doute d'admettre, sinon une réalité sensible ou idéale ${ }^{52} \mathrm{du}$ sens et des pensées, du moins un ordre logique des faits sociaux qui serait la condition de toute identification, dans l'intersubjectivité sociale, d'un contenu sémantique quelconque. Le sens et les pensées ne sont sans doute pas, comme le croyait Parsons, des variables indépendantes ou des objets susceptibles de lier par l'expérience nos variables propositionnelles, mais ce sont des conditions logiques qu'il est indispensable de prendre en compte dans toutes les opérations qui comportent des variables d'action ou d'institution. Cet ordre logique est évidemment

cédé, il n'est pas difficile d'inscrire toutes les variables intentionnelles, morales ou sociales, dans des propriétés qu'on applique ensuite à des êtres réellement existants tels que des agents individuels ou des actions physiques. Par exemple, sur les propriétés morales, on peut consulter J. Mc Dowell, Valeurs et qualités secondes, tr. fr. A. Ogien, in R. Ogien éd., Le réalisme moral, Paris, PUF, 1999, éd. orig. 1985. Le problème est seulement que ce procédé ne fait que reculer d'un cran la difficulté car, pour pouvoir faire des opérations sur les propriétés intensionnelles (avec un s, c'est-à-dire relatives au sens), il faut avoir un critère d'identification et de distinction qui, par définition, ne peut pas être strictement empirique, d'où les discussions sans fin des philosophes analytiques sur ce sujet.

51 W.V.O. Quine, Le mot et la chose, tr. fr. J. Dopp et P. Gochet, Paris, Flammarion, 1977, éd. orig. 1960.

52 Frege a tenté de donner une réponse circonstanciée au problème de l'objectivité du sens et des pensées, en les situant dans un troisième domaine qui ne serait réductible ni aux choses physiques, ni aux représentations psychologiques. Cette thèse n'est pas sans parenté avec la notion durkheimienne de réalité sociale sui generis qui, elle aussi, est distincte des faits physiologiques et psychologiques. Mais, comme l'ont souligné les critiques de Frege (cf. M. Dummett, The Social Character of Meaning, in Truth and other Enigmas, Cambridge, Harvard University Press, 1978, éd. orig. 1974), le caractère idéal du troisième domaine frégéen le prive aussi de toute réalité, à moins du supposer une existence idéale du sens et des pensées, ce qui ne va pas de soi. 
compatible avec le caractère aléatoire ou irrationnel d'un certain nombre d'événements sociaux, puisqu'il ne concerne pas le déroulement empirique des phénomènes, mais seulement la structure logique de leurs éléments constitutifs : actions, institutions, relations, affections, ou qualités. Mais c'est bien parce que nous possédons certains outils logiques et conceptuels généraux que nous pouvons différencier et identifier ces différents types d'éléments et établir des liens entre eux pour chaque situation particulière.

Par exemple, c'est parce que nous savons que les bâtiments urbains fixent la valeur de variables relatives à la propriété juridique que nous pouvons les mettre en relation avec des mouvements physique d'agents, eux-mêmes reconnus par exemple sous un certain sens du licite ou de l'illicite. Ou encore c'est parce que nous savons qu'un événement particulier, par exemple une déclaration publique, tombe sous un certain sens identique pour l'agent comme pour l'observateur, par exemple le sens d'une déclaration de guerre, que nous pouvons inscrire cet événement dans des chaînes causales impliquant d'autres événements. Ou enfin, c'est parce que nous supposons que des agents humains tiennent pour vraies ou valides certaines idées clairement identifiées, comme des idées protestantes ou antisémites, que nous pouvons expliquer leurs actions, et en particulier faire la part de ce qui, en elles, relève des idées et de ce qui n'en relève pas. Dans ces différents cas d'analyse empirique qui portent sur des objets physiques, des événements, des agents ou des actions, nous comptons toujours sur la puissance et la portée d'outils logiques communs pour ne pas douter que les agents soumis à l'analyse comprendront les faits que nous considérons à peu près de la même façon que nous, quelles que soient leurs singularités culturelles ou contextuelles. Et bien que les contenus de pensée ne soient pas vraiment des «variables indépendantes», nous nous en servons néanmoins comme moyen de spécifier les actions par rapport aux autres faits matériels pertinents et de repérer ainsi leur place dans des enchaînements causaux.

C'est cette ligne de réflexion qui m'a conduit, dans différents travaux ${ }^{53}$, à proposer de considérer le sens et les pensées comme l'effet, dans les comportements sociaux, de contraintes logico-sémantiques qui pèsent sur tout ce que les êtres humains ${ }^{54}$ peuvent accomplir et exprimer de sensé au cours de leur insertion dans le monde naturel. Cette idée peut être facilement illustrée par l'analyse du sens des actes sociaux: par exemple celui qui accomplit l'acte qui a le sens d'un compliment ou bien d'une promesse doit se plier à certaines contraintes conceptuelles, qui sont respectivement de dire du bien (et non du mal) de ce qui a été accompli par autrui (et non par soi-même) dans l'intérêt de celui qui reçoit le compliment (et non de celui qui l'émet); ou, pour la promesse, de s'engager sur (et non seulement d'annoncer) un acte futur de celui qui promet (et non pas de celui à qui on promet) favorable (et non pas nuisible) à celui à qui s'adresse la promesse ${ }^{55}$. D’une façon analogue quoique plus complexe, les idées calvinistes ou les idées

53 Cf. en particulier Sociologie de l' esprit, conceptualisation et vie sociale, Paris, PUF, 1997.

54 Et peut-être aussi d'autres êtres vivants comme des primates ou autres animaux.

55 Une analyse de ces contraintes conceptuelles peut évidemment toujours être contestée ou améliorée, - et je ne prétends pas avoir proposé, dans ce qui précède, une bonne théorie du compliment ou de la promesse -, mais il est difficile de nier que ces contraintes existent. 
antisémites dont il a été question plus haut, ne peuvent être repérées qu'à partir d'un certain nombre de règles logico-linguistiques qui permettent de différencier des contenus, ceux qui portent par exemple sur les voies du salut dans le cas des idées religieuses ou sur les qualités des groupes humains dans la cas d'une hiérarchie raciale.

Ce qu'on appelle le travail d'interprétation n'est d'ailleurs rien d'autre qu'un effort d'application à des cas concrets de ces outils logico-sémantiques dont sont équipés tous les sujets qui participent d'une langue naturelle, y compris bien sûr ceux qui, en tant que savants, exercent une profession qui consiste à ajuster leurs pensées aux règles logico-sémantiques les plus clairement répertoriées dans l'ensemble du monde social. Un théoricien de l'interprétation tel que D. Davidson s'est d'ailleurs inspiré de considérations analogues pour rendre compte de la compréhension et de la traduction interculturelle. Davidson substitue en effet à la thèse plutôt pessimiste de Quine sur l'indétermination de la traduction, une thèse beaucoup plus optimiste de l'interprétation radicale ${ }^{56}$ qui cherche à rendre compte du fait, malgré tout indiscutable, de la traduction interlinguistique ou interculturelle, fût-elle approximative. L'idée de Davidson est que l'interprétation interlinguistique et interculturelle est rendue possible à la fois par des principes de vérité qui permettent aux êtres humains de partager un grand nombre de croyances communes relatives au monde et un principe de rationalité qui nous fait supposer, jusqu'à preuve du contraire, qu'il doit y avoir des raisons intelligibles dans les discours ou les actes des autres sujets ${ }^{57}$.

L'hypothèse d'un ordre logique du sens et des pensées qui structure les faits sociaux, ou en résumé d'un ordre sémantique contraignant pour tous les êtres qui veulent participer à des coordinations par le sens, et non pas seulement par des stimulations sensibles, n'est, au fond, qu'une sorte d'explicitation logique de la thèse durkheimienne de la réalité sociale sui generis. C'est en effet la conjonction des contraintes liées à une coordination par le sens et des contraintes liées à l'objectivité du monde et des objets dans lesquels vivent les êtres humains qui instaure la contrainte sémantique dont il est question, laquelle se manifeste aux êtres sociaux sous la forme d'une norme qui est à la fois logique et naturelle - et qui peut prendre évidemment toutes sortes de formes culturelles. Dans un monde où les choses sont ce qu'elles sont indépendamment de l'opinion qu'on en a, il n'est pas possible de se coordonner par un langage qui fait référence à ces choses sans se plier à une norme de vérité qui, même lorsqu'elle est violée, est le seul moyen

56 L'interprétation radicale, in Enquête sur la vérité et l'interprétation, tr. fr. P. Engel 1993, Nîmes, Jacqueline Chambon, 1967, éd. orig. 1973.

57 Formellement, Davidson utilise le schéma de la théorie de la vérité élaboré par A. Tarski ( $L a$ conception sémantique de la vérité et les fondements de la sémantique, tr. fr. G. Grangier et al., in Logique, sémantique, métamathématiques, t. 2, Paris, A. Colin, 1974, éd.. orig. 1944) pour rendre compte de l'impossibilité logique d'inclure sans contradiction un prédicat de vérité dans un langage formalisé. La solution envisagée par Tarski consistait à inclure le prédicat de vérité dans un langage de rang supérieur de telle sorte qu'une phrase «s $s$ du langage objet est vraie si et seulement si il est possible, dans un métalangage, d'énoncer s. Davidson s'inspire de ce résultat pour supposer que, dans une situation dite d'interprétation radicale, c'est-à-dire une situation où l'on n'a aucun rudiment de la langue de l'étranger, une phrase inconnue « s » est vraie si et seulement si on peut lui trouver un certain équivalent s dans sa propre langue, par exemple « es regnet » est vrai si et seulement s'il pleut. 
d'assurer la continuité sensée de cette coordination. La prédication, les fonctions de vérité et la quantification qui sont, selon Quine ${ }^{58}$, les constructions de base de la logique sont aussi les opérations de base de la communication sociale ordinaire qui, quelle que soit la multiplicité de ses formes et de ses effets pragmatiques, repose aussi sur des assumptions existentielles susceptibles d'être vérifiées ou validées par les partenaires d'interaction. Et l'ajustement fonctionnel des pratiques réciproques qui, dans la vie sociale courante, tend à s'établir sur la base des réactions des partenaires, doit le caractère particulier qu'il prend chez les humains au fait que ces réactions ne sont pas fondées uniquement sur des stimulations sensibles mais aussi sur la satisfaction de variables propositionnelles - par exemple, dans une société civilisée, l'ajustement des partenaires ne se fait pas seulement par des gifles ou des caresses, mais aussi par des expressions articulées qui permettent à un sujet de penser que son partenaire a ou non tel ou tel type d'attente ou d'attitude à son égard.

On peut, à partir de là, concevoir l'idée d'un programme sémantique ${ }^{59}$ pour la sociologie, au sens d'une étude générale des relations entre les pensées des sujets sociaux et leurs objets, et en particulier l'étude des conditions conceptuelles susceptibles de rendre ces pensées vraies ou fausses - autrement dit, il s'agit de rechercher les contraintes sémantiques qui peuvent assurer à un événement ou un objet social le sens qu'il est supposé avoir. La vérité des propositions sociologiques qui portent sur le sens, les pensées, les motifs, les valeurs, dépend en effet de leur capacité à identifier correctement les contraintes logico-sémantiques qui pèsent sur les sujets sociaux soumis à l'analyse. On a dans ce cas une situation de redondance logique qui fait dépendre la valeur de vérité des propositions, non pas seulement d'une relation directe à certains états de choses, mais d'une relation oblique ayant comme intermédiaire les pensées des sujets sociaux sur les états de chose considérés. Nous disons par exemple que $\mathrm{X}$ promet ou se marie, non pas seulement parce qu'on le voit directement émettre certains bruits ou faire certains mouvements dans certains lieux, mais parce qu'on lui prête, ainsi qu'à son entourage, des pensées pourvues d'une certaine structure conceptuelle qui sont la condition pour que sa promesse ou son mariage soient ce qu'ils sont.

On dira, plus généralement, que dans une description sociologique qui implique un sens ou une pensée, la relation entre la description (par exemple X fait un reproche à $\mathrm{Y}, \mathrm{X}$ est candidat à la députation, $\mathrm{X}$ se suicide, $\mathrm{X}$ se fait passer pour une femme, $X$ est protestant, $X$ est antisémite, etc.) et ce qui se produit, ne peut

58 Le mot et la chose, op. cit., p. 316.

59 Pour le logicien A. Tarski, « la sémantique est une discipline qui, en termes généraux, traite de certaines relations entre les expressions d'un langage et les objets (ou «états de choses») auxquels se réfèrent ces expressions » (La conception sémantique de la vérité, op. cit., p. 273). Et Tarski poursuivait en donnant comme exemples typiques de concepts sémantiques les concepts de désignation, de satisfaction, de définition, ainsi que le concept de vérité, qui bien que n'étant pas luimême un concept relationnel, se définit par un concept relationnel qui est la satisfaction. Tarski remarque aussi que « les notions sémantiques se rencontrent, en nombre plus ou moins grand, en psychologie, en sociologie et pratiquement dans toutes les sciences humaines » (ibid., p. 299). Il ajoute enfin qu' « il est peut-être superflu de dire que la sémantique ne peut pas trouver d'application dans les sciences naturelles telles que la physique, la biologie, etc. Car dans aucune de ces sciences nous n'avons affaire à des phénomènes linguistiques ni même à des relations sémantiques entre les expressions linguistiques et les objets qu'elles concernent.» 
être satisfaite que si elle inclut comme une de ses composantes la relation entre la pensée du sujet observé et ce qui se produit. Un programme sémantique en sociologie consiste alors à explorer l'ensemble des moyens logiques et conceptuels permettant cette inclusion du sens et des pensées dans les faits sociaux ${ }^{60}$. Autrement dit, on étudie les contraintes sémantiques qui pèsent sur les différents concepts qui se rapportent aux faits sociaux pour savoir s'il peut être vrai que l'agent a agi sous cette description, c'est-à-dire avec les pensées correspondantes. Par exemple, si on veut savoir si quelqu'un s'est suicidé, on s'interroge sur ce qu'il a fait en fonction des pensées qu'on prête normalement à un suicidé, et en particulier celle de vouloir mourir. C'est du reste ce que chacun d'entre nous fait tous les jours pour savoir ce qui se passe autour de lui, sauf que les contraintes qu'il admet pour les concepts qu'il utilise n'ont pas la précision qu'on est en droit d'attendre d'une recherche sociologique sur le mariage, le mensonge ou la flatterie.

On retrouve ici le schéma d'interprétation radicale de Davidson, qui suppose l'appui sur une vérité supposée prévalante comme moyen de comprendre le sens de l'activité ou du langage d'autrui et qui est en fait une procédure très courante de la vie sociale, et pas seulement dans les échanges interculturels. Ce qui caractérise cette méthode sémantique ordinaire d'interprétation, ce ne sont pas seulement des hypothèses sur les valeurs de vérité des phrases que l'on énonce, mais aussi des hypothèses sur les phrases qu'on devrait prononcer ou sur les actes qu'on devrait ou, au moins, qu'on ne devrait pas accomplir compte tenu de la situation. L'analyse sociologique et historique est elle-même fondée la plupart du temps sur ce modèle formel de comparaison des données factuelles à des virtualités normatives, car il paraît difficile de comprendre les actions d'autrui sans envisager soi-même ce qui serait possible ou même préférable dans la situation considérée. C'est en ce sens qu'on peut dire que l'analyse sociologique, aussi longtemps qu'elle prend au sérieux le programme wébérien de compréhension du sens de l'action, comporte une dimension normative qui consiste à faire des hypothèses sur le sens qui devrait prévaloir dans une situation pour pouvoir comprendre celui qui prévaut en réalité. Les cas d'anomies et de pratiques extrêmes sont du reste particulièrement révélateurs de cette démarche logico-normative, bien qu'ils n'en épuisent pas la portée.

\section{CNRS-CERSES}

60 Mon ouvrage sur Le sens de la justice, essais de sémantique sociologique, Paris, PUF, 2001, présente un certain nombre de travaux pratiques dans cette direction, à partir des concepts de justice, de contrat, d'ordre, de louange et d'accueil. 